\title{
A Target Site for Spontaneous Insertion of IS10 Element in pUC19 DNA Located within Intrinsically Bent DNA
}

\author{
Shungo Kobori ${ }^{1}$, Yumi $\mathrm{Ko}^{2}$ and Mikio Kato ${ }^{1, *}$ \\ ${ }^{I}$ Department of Biological Science, Graduate School of Science, and ${ }^{2}$ Department of Earth and Life Science, College of \\ Integrated Arts and Sciences, Osaka Prefecture University, 1-1 Gakuencho, Sakai 599-8531, Japan
}

\begin{abstract}
Residual insertion sequence elements (IS elements) in Escherichia coli strains that are commonly used for DNA cloning are known to cause cloning artifacts by transposing themselves into the recombinant DNA fragments. In such cases, chance insertion of $I S$ elements may occur at integration sites in the cloning targets, which in the case of the $I S 10$ element is a 9-bp consensus sequence. We report here that the integration of ISIO-related DNA sequences into the pUC19 cloning vector and its derivative occurred with considerable frequency in E. coli strains JM107 and DH10B, with duplication of a 9-bp segment (TCTAAAGTA). Notably, native polyacrylamide gel electrophoresis revealed that intrinsically bent DNA flanks the insertion site.
\end{abstract}

Keywords: Bent DNA, cloning vector, DNA curvature, IS10, mobile element.

\section{INTRODUCTION}

Transposition of $I S$ elements is a strong motive force underlying genetic mutations in Escherichia coli [1] and affects some mobile elements, even in resting cells [2]. Kovarík et al. [3] reported that ISIO-related sequences are major contaminants of the cloned DNA sequences appearing in international DNA databases (EMBL, GenBank, and DDBJ). For instance, they showed that a $1.3-\mathrm{kb} I S 10$-related DNA fragment was inserted into the recombinant plasmid during recloning of the repetitive sequence R8.3 from Nicotiana tabacum. It also has been reported that cloning eukaryotic DNA enhances transposition of $I S$ elements [4], and that transposition of $I S 2$ element into the cloning vector pUC18 can occur spontaneously [5].

DNA conformation is known to be a key determinant modulating genome function [6]. DNA bending, or curvature, is one of the unusual DNA conformations that can affect transcription in both eukaryotes and prokaryotes [7, 8]. Bent DNA is also involved in regulating DNA recombination [9-11]. In the present paper, we report spontaneous insertion of IS10 element into the plasmid vector pUC19 and its derivative, pAL79, at a site within an intrinsically bent DNA region.

\section{EXPERIMENTAL RESULTS AND DISCUSSION}

During the course of analyzing the topology of supercoiled plasmid DNA of pUC19 and its derivatives, we came across several topoisomers of covalently closed circular DNA (cccDNA), the sizes of which were unexpected (data not shown). These unexpected spots of DNA topoisomer

*Address correspondence to this author at the Department of Biological Science, Graduate School of Science, Japan; Tel/Fax: +81-72-254-9746;

E-mail:mkato@b.s.osakafu-u.ac.jp,mikio_kato@mac.com appeared on 2-dimensional agarose gel electrophoresis when the DNA samples were prepared from old cultures (e.g., cultures left at room temperature for several days after the stationary phase). To identify DNA molecules of unexpected size, we examined E. coli strain JM107 transformed using conventional procedures with plasmid pAL79, a pUC19 derivative containing the AL79 minisatellite [12]. The transformants were collected and inoculated into $2 \mathrm{ml}$ of LB medium containing $50 \mu \mathrm{g} / \mathrm{ml}$ of ampicillin, after which they were incubated at $37^{\circ} \mathrm{C}$ overnight with shaking. The plasmid DNA was then isolated from $0.5 \mathrm{ml}$ of culture using the rapid boiling method [13] and electrophoresed on agarose gel, which confirmed that the cells contained authentic pAL79 plasmid by size estimation. The overnight cultures were then left for 10 days at room temperature, after which aliquots were spread on ampicillin $(50 \mu \mathrm{g} / \mathrm{ml})$-containing LB agar plates. After incubating overnight, 109 colonies were collected, irrespective of their size, and each was inoculated into $2 \mathrm{ml}$ of LB medium containing $50 \mu \mathrm{g} / \mathrm{ml}$ of ampicillin. The plasmid DNA was again isolated using the boiling method, and the electrophoretic patterns were examined. We found that five of the plasmid DNA samples showed an additional DNA band (an example is shown in Fig. 1A), which we hypothesized might be the result of spontaneous insertion of an $I S$ element, as suggested by de Togni et al. [5]. But because the recA-genotype is known to affect the transposition efficiency of $I S$ elements [14-16], in subsequent experiments we examined the occurrence of altered plasmid molecules using Escherichia coli strain DH10B, a recA-deficient strain. With the same subculture procedure, one of 296 colonies of DH10B transformants retained the altered pAL79 plasmid (Fig. 1B, vertical arrow), while 2 out of 100 colonies of DH10B transformants retained the altered pUC19 plasmid (Fig. 1C). While the majority pAL79 DNA was normal in the fresh overnight cultures of JM107 transformants harboring the altered pAL79 (Fig. 1A), the altered 


\section{(A) \\ (B)}

$12 \mathrm{M}$

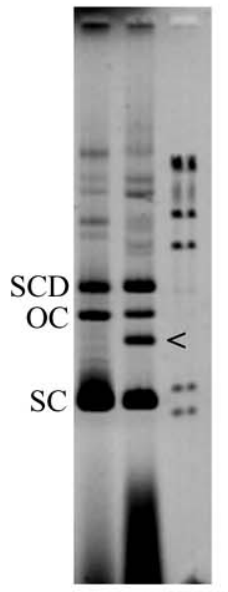

pAL79/JM107

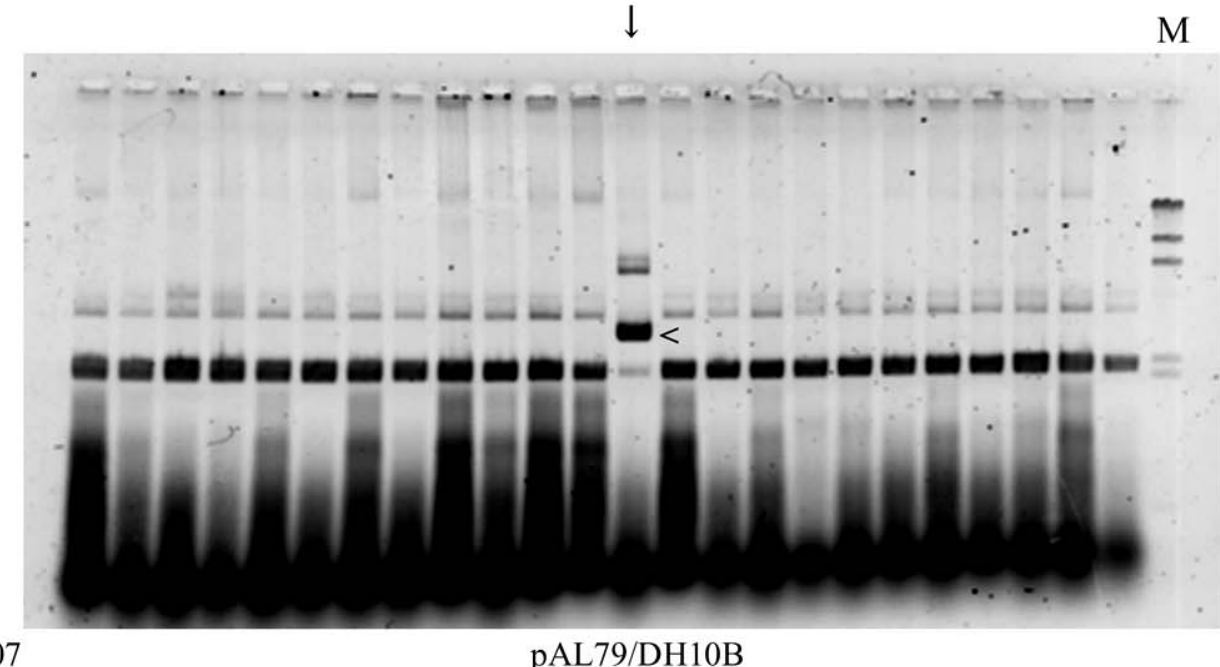

pAL79/DH10B
(C)
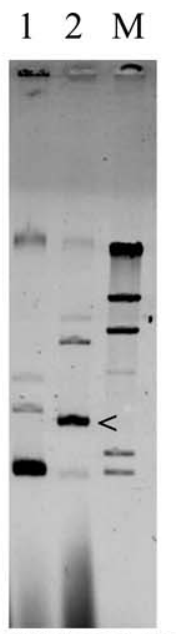

pUC19/DH10B

Fig. (1). Electrophoretic mobility of representative plasmid DNA isolated from overnight cultures. Plasmid DNA isolated from $E$. coli transformants using the rapid boiling method [13] was fractionated on an agarose gel. Because the samples were not treated with RNaseA, RNA appeared at the bottom of the gel. Horizontal arrowheads $(<)$ indicate the plasmid DNA showing size alteration. (A) pAL79 DNA isolated from JM107 transformants. Lane 1, sample containing normal pAL79 plasmid; lane 2, sample containing altered plasmid; lane M, HindIII-digested $\lambda$ phage DNA size markers. SC, supercoiled molecule; OC, open circle (relaxed molecule); SCD, supercoiled dimer molecule. (B) pAL79 DNA isolated from DH10B transformants. Each lane corresponds to a DNA sample isolated from an independent colony. The vertical arrow indicates the sample containing the altered plasmid DNA. Lane M, HindIII-digested $\lambda$ phage DNA size markers. (C) pUC19 DNA isolated from DH10B transformants. Lane 1, sample containing normal pUC19 plasmid; lane 2, sample containing altered plasmid; lane M, HindIII-digested $\lambda$ phage DNA size markers.

plasmid was more abundant than the normal plasmid in DH10B transformants harboring pAL79 (Fig. 1B) or pUC19 (Fig. 1C). At this moment, it is unclear why the relative amount of altered size plasmid varies with the host strain (JM107 or DH10B). High copy number of the plasmid DNA in the cells, low rate of IS transposition and the recA genotype may be the factors that relate to this observation.

To characterize the altered plasmid DNA, the fraction containing the altered pAL79 (marked with an arrowhead in the lane 2 of Fig. 1A) was recovered from the agarose gel and used to transform E. coli strain DH10B. The recloned pAL79 cccDNA was then isolated from the transformants, and digested using the restriction endonuclease Dra I. This indicated that a DNA sequence was inserted into the $0.7-\mathrm{kb}$ Dra I fragment of pUC19 vector, resulting in the presence of an additional DNA fragment and making the 0.7-kb Dra I fragment slightly longer (Fig. 2A). We then carried out PCR amplification with several sets of primers to identify the inserted fragment. We eventually determined that the insertion had occurred near the upstream terminus of the 0.7kb Dra I fragment, after which we amplified the 267-bp region spanning positions 1691 to 1957 of pUC19 (GenBank accession M77789) using the following primers: 5'TGAACGAAATAGACAGATCGCTGAGAT-3'(forward) and 5'-GTTTGCAAGCAGCAGATTACGCGCAGA-3' (reverse). When the recloned cccDNA (altered pAL79) was used as the PCR template, a 1.6-kb fragment was amplified (Fig. 2B, lane 2). But when plasmid preparations from DH10B (the lane marked with an arrow in Fig. 1B and lane 2 of Fig. 1C served as the template, a $1.6-\mathrm{kb}$ fragment in addi- tion to the authentic 267-bp fragment was amplified (data not shown).

The nucleotide sequences of the 1.6-kb fragments from both the altered pAL79 and pUC19 (from DH10B transformants) plasmids were determined after their purification from the excised gels. We found that the inserted sequences shared about $94 \%$ identity with most ISIO elements and that the insertion into pAL79 and pUC19 occurred at the same site: position 1771-TACTTTAGA-1779 in the vector region of pUC19 with duplication of a 9-bp segment. Although the nucleotide sequences differed to a small degree (94\% identity) from that reported by Kovarík et al. [3], the inserted part of the ISIO element (corresponding to the region spanning nucleotides 58 to 1390 of AJ293237) was nearly identical except the boundary nucleotides.

The observed target site for IS10 insertion did not completely match the consensus sequence for IS10 integration sites (5'-NPuCNNNGPyN-3') reported by Kovarík et al. [3]. Instead, the IS10 insertion into pUC19 DNA may be related to an intrinsic preference for the DNA conformation. For that reason, we compared the electrophoretic mobility of DNA fragments containing the ISIO target site in their native conformations in $5 \%$ polyacrylamide gels at $4^{\circ} \mathrm{C}$. Three different 257-bp fragments were prepared by PCR with the appropriate primers and subjected to electrophoresis. The results of the PCR are shown in Fig. (3) and the physical map of the target regions is shown in Fig. (4A and $\mathbf{4 B}$ ). The fragment containing the IS10 target site in its middle (Fig. (3), lane 1) migrated more slowly than the others, suggesting it assumes a bent conformation, as bending in the middle of the fragment would cause a more pronounced retardation of mobility 
(A)

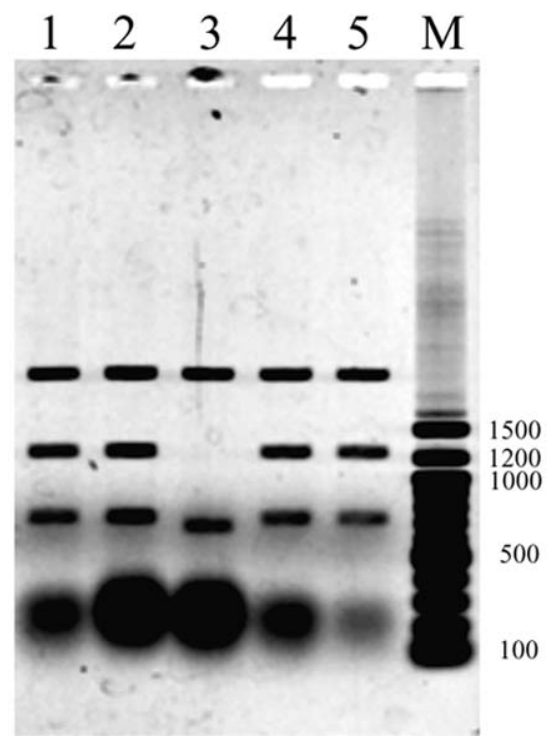

(B)

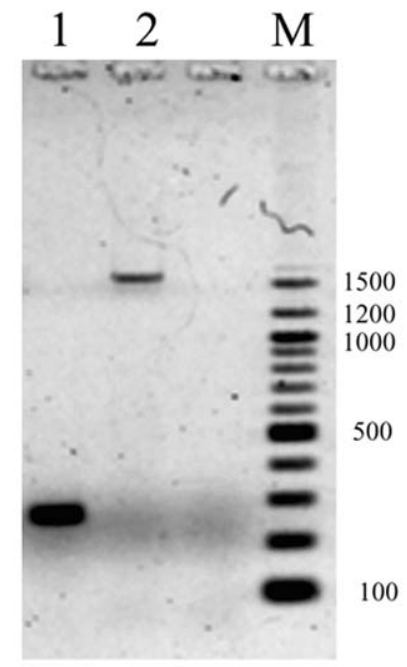

Fig. (2). Restriction analysis and PCR amplification using pAL79 DNA containing an insertion as a template. (A) Dra I digestion of recloned pAL79. Recloned DNA samples were prepared from 4 independent colonies, and both altered and control plasmids were examined. Lanes 1, 2, 4 and 5, Dra I-digested recloned altered pAL79; lane 3, Dra I-digested control pAL79; lane M, 100-bp DNA ladder size markers. The large blobs at the bottom are RNA that remained in the plasmid preparation. (B) Electrophoretic mobility of PCR-amplified fragments. Lane 1, normal control pAL79 used as a template; lane 2, altered pAL79 used as a template; lane M, 100-bp DNA ladder size markers.

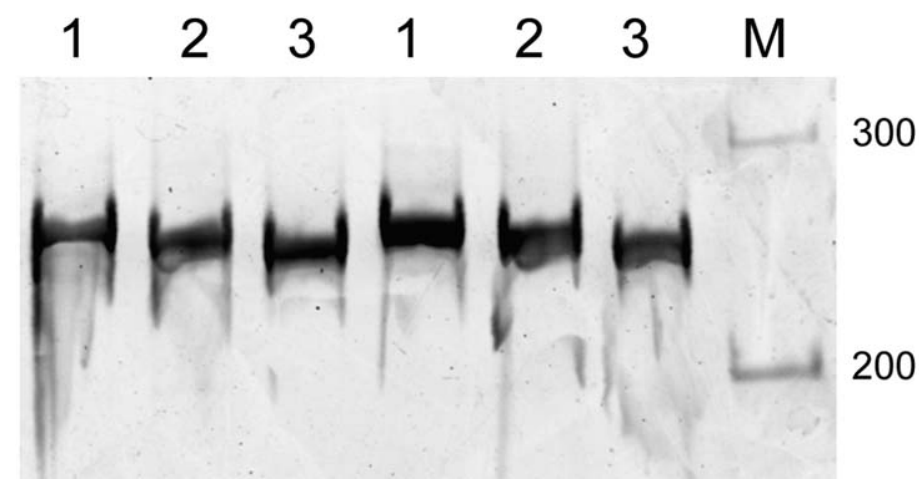

Fig. (3). Native polyacrylamide gel electrophoresis of DNA fragments containing an ISI0 target site. DNA fragments obtained by PCR amplification were electrophoresed on native $5 \%$ polyacrylamide gel at $4^{\circ} \mathrm{C}$. Lane 1, a 257-bp fragment spanning positions 1661 to 1917 of pUC19; lane 2, a 257-bp fragment spanning postions1621 to 1877 of pUC19; lanes 3, a 257-bp fragment spanning positions 1541 to 1797 of pUC19; lanes M, 100-bp DNA ladder size markers. Because the difference in the electrophoretic mobility was subtle, DNA samples were applied to the gel in duplicate. The respective DNA primer pairs used for the PCR were: 1661-CTACACGACGGGGAGTCAGGCAA CTAT (forward) and 1917-AAGAAGATCCTTTGATCTTTTCTACGG (reverse); 1621- CACTGGGGCCAGATGGTAAGCCCTCCC (forward) and 1877- GTGGAACGAAAACTCACGTTAAGGGAT (reverse); and 1541- GCGCTCGGCCCTTCCGGCTGGCTGGTT (forward) and 1797- AATGAAGTTTTAAATCAATCTAAAGTA (reverse). The IS10 target site is 1771-TACTTTAGA-1779 of pUC19.

than bending elsewhere [6]. In similar fashion, integration of IS2 into the lac operon promoter in pUC18 [5] also might reflect the flexibility of the target DNA region, as the lac promoter region is reportedly looped as a result of DNA bending $[17,18]$. To support DNA bending occurring near the integration site 1771-TACTTTAGA-1779, we have computed the predicted curvature and bendability by Bend.it server [19]. The curvature prediction has found several local maximum points, and one of the curvature peaks appears coincidence with a local minimum of the bendability, where it flanks the integration site (Fig. 4C, around the nucleotide position 1791). This suggests that the rigid bent helical structure is formed at the local maximum peak of predicted curvature near the integration site. Other curvature peaks exist in relatively higher bendability regions meaning that the regions are curved but rather flexible.

Not only does transposition often occur spontaneously in resting cells [2], it can be induced by UV irradiation [20], and the IS10 element is a major contaminant of eukaryotic DNA sequences in databases [3]. It is known that insertion of sequence elements is intrinsically regulated by transcriptional, translational and pos-translational mechanisms, as well as by host factors [21]. However, the DNA conformation at the target site may also affect the efficiency of transposition, since it modulates various other processes affecting DNA (transcription, replication and recombination) [6]. And also, as reported by Nash and colleagues [22, 23], bending 
(A)

1541 gcgctcggeccttccggctg

1561 gctggtttat tgctgataaa tctggagccg gtgagcgtgg gtctcgcggt atcattgcag

1621 cactggggcc agatggtaag ccctcccgta tcgtagttat ctacacgacg gggagtcagg

1681 caactatgga tgaacgaaat agacagatcg ctgagatagg tgcctcactg attaagcatt

1741 ggtaactgtc agaccaagtt tactcatata TACTTTAGAt tgatttaaaa cttcattttt

1801 aatttaaaag gatctaggtg aagatccttt ttgataatct catgaccaaa atcccttaac

1861 gtgagttttc gttccactga gcgtcagacc ccgtagaaaa gatcaaagga tcttctt 1917

(B)

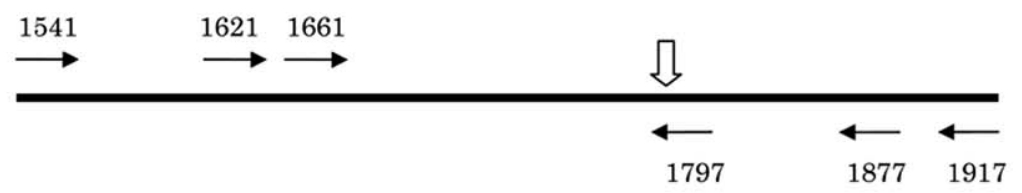

1

2
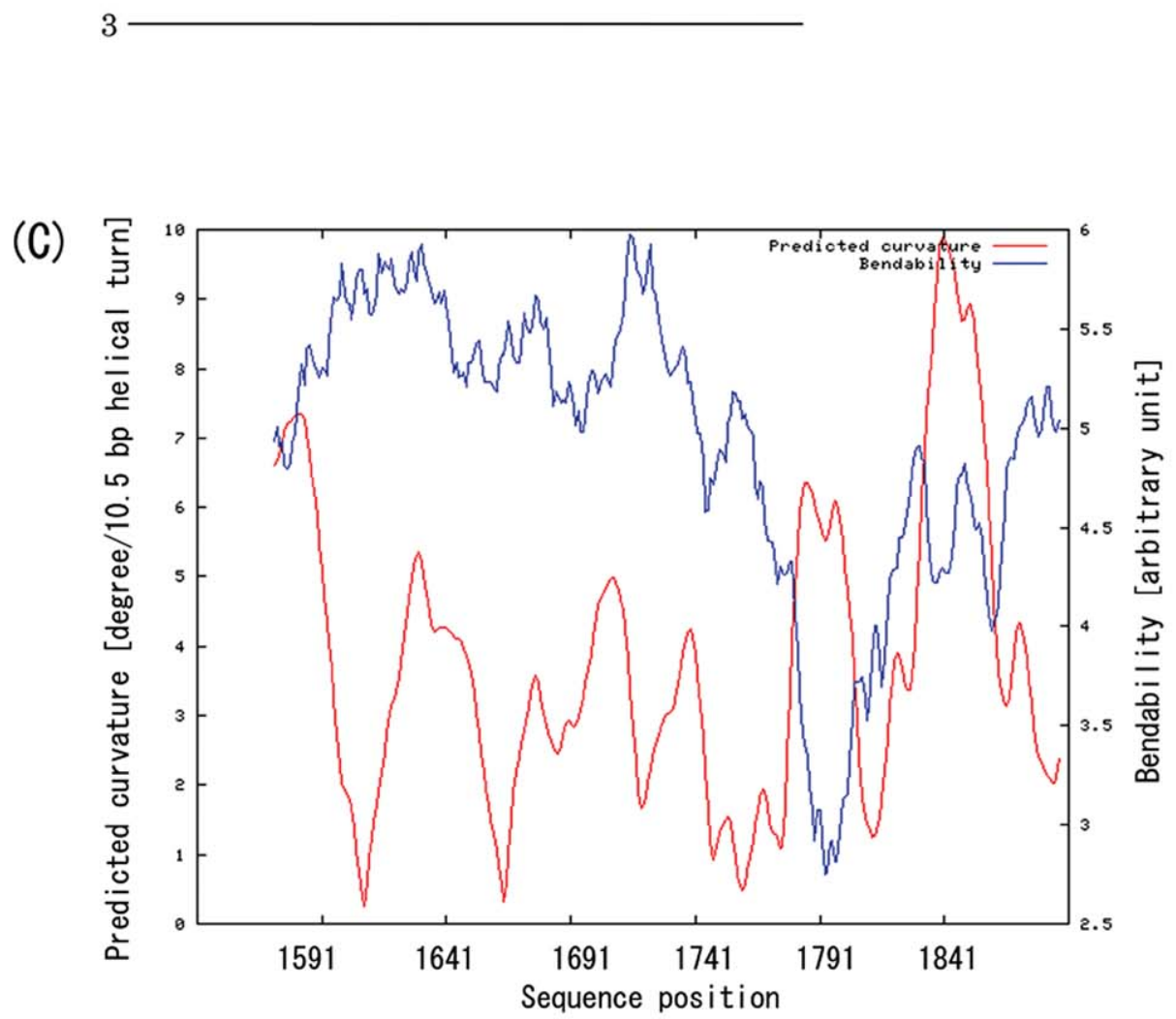

Fig. (4). Schematic representation of the ISI0 target region. (A) Nucleotide sequence of the IS10 target region in pUC19 vector. The integration site (9-bp segment) is indicated by capitals, and two closely located Dra I restriction sites are underlined. (B) Map of the PCRamplified fragments. Horizontal arrows labeled with nucleotide positions indicate the location of the 5'-termini of the PCR primers, and the vertical open arrow indicates the site of IS10 insertion. Thin horizontal lines labeled 1-3 show the PCR-amplified fragments fractionated by native polyacrylamide gel electrophoresis (Fig. 3). (C) Predicted curvature and bendability. The bendability/curvature propensity plots were calculated with the bend.it server [19] using the DNase I based bendability parameters of Brukner et al. [25] and the consensus bendability scale [26]. Bendability was plotted in cyan, and the predicted curvature was plotted in magenta. 
of DNA at bacteriophage $\lambda$ attachment site has occurred in the integrative recombination. Consistent with that idea, the native polyacrylamide gel electrophoresis carried out in the present study showed that the site targeted by the ISIO element is surrounded by intrinsically bent DNA. Given that bent DNA segments tend to preferentially locate at the apices of plasmid superhelices [24], it may be that the bent DNA region surrounding the $I S 10$ target site is situated at the apex of a plectonemic superhelix, thereby facilitating ISIO insertion.

\section{CONCLUSION}

In summary, we report here on the susceptibility of a plasmid vector to insertion of an IS10 element. Insertion of IS10 element into a plasmid vector has been shown to occur spontaneously during storage of stationary cultures of $E$. coli strains JM107 and DH10B. At present, it remains unclear whether any other insertions into the plasmid occur in E. coli.

\section{ABBREVIATION}

cccDNA $=$ Covalently closed circular DNA

\section{REFERENCES}

[1] Rodriguez H, Snow ET, Bhat U, Loechler EL. An Escherichia coli plasmid-based, mutational system in which supF mutants are selectable-insertion elements dominate the spontaneous spectra. Mutat Res 1994; 270: 219-31.

[2] Naas T, Blot M, Fitch WM, Arber W. Insertion sequence-related genetic variation in resting Escherichia coli K-12. Genetics 1994; 136: 721-30.

[3] Kovarík A, Matzke MA, Matzke AJ, Koulaková B. Transposition of IS10 from the host Escherichia coli genome to a plasmid may lead to cloning artifacts. Mol Genet Genomics 2001; 266: 216-22.

[4] Amster O, Salomon D, Zamir A. A cloned immunoglobulin cDNA fragment enhances transposition of $I S$ elements into recombinant plasmids. Nucleic Acids Res 1982; 10: 4525-41.

[5] de Togni P, Fox ES, Morrissey S, Babior BM, Levy SB. Spontaneous insertion of an IS2 element into the promoter region of the lac operon. Plasmid 1988; 20: 143-7.

[6] Sinden RR. DNA Structure and function. San Diego: Academic Press 1994.

[7] Pérez-Martín J, Rojo F, de Lorenzo V. Promoters responsive to DNA bending: a common theme in prokaryotic gene expression. Microbiol Rev 1994; 58: 268-90.

[8] Ohyama, T. DNA conformation and transcription. Georgetown: Landes Bioscience 2005.
[9] Goodman SD, Nash HA. Functional replacement of a proteininduced bend in a DNA recombination site. Nature 1989; 341: 2514.

[10] Milot E, Belmaaza A, Wallenburg JC, Gusew N, Bradley WEC, Chartrand P. Chromosomal illegitimate recombination in mammalian cells is associated with intrinsically bent DNA elements. EMBO J 1992; 11: 5063-70

[11] Hallet B, Rezsöhazy R, Mahillon J, Delcour J. IS231A insertion specificity: consensus sequence and DNA bending at the target site. Mol Microbiol 1994; 14: 131-9.

[12] Elmesiry GE, Okai S, Hokabe S, et al. Isolation and characterization of simple repeat sequences from the yellow fin sea bream Acanthopagrus latus (Sparidae). Mol Biol Rep 2005; 32: 117-6.

[13] Holms DS, Quigley M. A rapid boiling method for the preparation of bacterial plasmids. Anal Biochem 1981; 114: 193-7.

[14] Skaliter R, Eichenbaum Z, Shwartz H, Ascarelli-Goell R, Livneh Z. Spontaneous transposition in the bacteriophage 1 cro gene residing on a plasmid. Mutat Res 1992;267: 139-51.

[15] O'Neil JP, Bogosian G. Residual IS10 elements do not exhibit transpositional mutagenesisin Escherichia coli recA mutants: studies with strains DH5 $\alpha$ and DH10B. FOCUS 1994; 16: 106-8.

[16] Eichenbaum Z, Livneh Z. Intermolecular transposition of IS10 causes coupled homologous recombination at the transposition site. Genetics 1995; 140: 861-74.

[17] Buckle M, Buc H, Travers AA. DNA deformation in nucleoprotein complexes between RNA polymerase, cAMP receptor protein and the lac UV5 promoter probed by singlet oxygen. EMBO J 1992; 11:2619-25.

[18] Becker NA, Kahn JD, Maher LJ 3rd. Effects of nucleoid proteins on DNA repression loop formation in Escherichia coli. Nucleic Acids Res 2007; 35: 3988-4000.

[19] Bend.it server. (accessed 26 June, 2009) http://hydra.icgeb.trieste.it/ dna/bend_it.html

[20] Eichenbaum Z, Livneh Z. UV light induces IS10 transposition in Escherichia coli. Genetics 1998; 149: 1173-81.

[21] Mahillon J, Chandler M. Insertion Sequences. Microbiol Mol Biol Rev 1998; 62: 725-74.

[22] Robertson CA, Nash HA. Bending of the bacteriophage $\lambda$ attachment site by Escherichia coli integration host factor. J Biol Chem 1988; 263: 3554-57.

[23] Goodman SD, Nicholson SC, Nash HA. Deformation of DNA during site-specific recombination of bacteriophage $\lambda$ : Replacement of IHF protein by HU protein or sequence-directed bends. Proc Natl Acad Sci USA 1992; 89: 11910-4.

[24] Vologodskii AV, Zhang W, Rybenkov VV, et al. Mechanism of topology simplification by type II DNA topoisomerases. Proc Natl Acad Sci USA 2001; 98: 3045-9.

[25] Brukner I, Sanchez R, Suck D, Pongor S. Sequence dependent bending propensity of DNA as revealed by DNAse I: Parameters for trinucleotides. EMBO J 1995; 14: 1812-8.

[26] Gabrielian A, Pongor S. Correlation of intrinsic DNA curvature with DNA property periodicity. FEBS Lett 1996; 393: 65-8. 